\title{
ARQ Protocols in Cognitive Decode-and-Forward Relay Networks: Opportunities Gain
}

\author{
Zongsheng ZHANG ${ }^{1,2}$, Qihui $W U^{1}$, Jinlong $W A N G^{1}$ \\ ${ }^{1}$ College of Communications Engineering, PLA University of Science and Technology, Street YuDao, Nanjing, China \\ ${ }^{2}$ College of National Information Science, JieFangGongyuan Road, Wuhan, China
}

zhangzongsheng1984@163.com, 532692271@qq.com, 15951803143@163.com

\begin{abstract}
In this paper, two novel automatic-repeat-request (ARQ) based protocols are proposed, which exploit cooperation opportunity inherent in secondary retransmission to create access opportunities. If the signal was not decoded correctly by destination, another user can be acted as a relay to reduce retransmission rounds by relaying the signal. For comparison, we also propose a Direct ARQ Protocol. Specifically, we derive the exact closed-form outage probability of three protocols, which provides an effective means to evaluate the effects of several parameters. Moreover, we propose a new metric to evaluate the performance improvement for cognitive networks. Finally, Monte Carlo simulations were presented to validate the theory analysis, and a comparison is made among the three protocols.
\end{abstract}

\section{Keywords}

Cognitive relay networks, outage probability, automatic-repeat-request

\section{Introduction}

Since the 1920s, each wireless communications system has been required to have an exclusive license from government in order to mitigate the interference from each other. Over the past decades, we have witnessed an increasing development and popularity of wireless communications, which has turned the limited spectrum resources into a scarce resource, which consequently imposes increasing stress on the fixed and limited radio spectrum. In practice, recent researches by the Federal Communications Commission (FCC) indicated that the utilization of spectrum is very low varying form $6 \%$ to $85 \%$, whereas only $2 \%$ of spectrum would be used in the US at any given moment [1]. The inefficient use of the radio spectrum resources under the current fixed policy, as well as the demand for more and better services has contributed to the reconsideration of the way the spectrum is used today and has led to decision of the FCC to allow access of unlicensed users to the broadcast television spectrum at locations where that spectrum is not being used by licensed users [2].
In order to increase the efficiency of spectrum utilization, diverse types of technologies have been deployed. The key technology towards efficient spectrum usage is Cognitive Radio, which was first introduced by J. Mitola III [3]. Cognitive radio is typically built on the software-defined radio technology, in which the transmitter's operating parameters, such as the frequency range, modulation type, and maximum transmission power can be dynamically adjusted by software [4]- [7]. Cognitive radio allows unlicensed users to access licensed users free from harmful interference. Specifically, the unlicensed (Secondary) users can access the spectrum resources originally licensed to primary users through spectrum overlay, interweave, and underlay ways. In overlay spectrum way, the unlicensed users need allocate a fraction of resources to maintain or improve the transmission of the primary users using sophisticated signal processing and coding. In interweave spectrum way, the unlicensed users share the licensed spectrum when the spectrum is idle, known as spectrum hole. Contrary to overlay and interweave ways, the unlicensed users control their transmit power to satisfy interference power constraint which licensed user can tolerate in underlay way. Due to its simplicity, we focus on underlay spectrum sharing in this paper.

Direct transmission, which demands large transmit power, ends up with small opportunity of access and hence low spectrum sharing efficiency. At the same time, cooperative diversity [8] has emerged as a promising technique to combat fading in wireless communications. Therefore, cognitive radio combined with cooperative diversity technique, referred to as cognitive relay networks (CRNs) [9][10], appears as an attractive solution to boost the spectrum sharing efficiency. In [9], a linear cooperative sensing frame work has been proposed based on the combination of local statistics from individual cognitive users. A distributed algorithm for channel access and power control was proposed for cognitive multi-hop relays in [10]. In [11], the authors have considered the application of cooperative diversity to spectrum sensing, and shown that the sensing performance is improved by exploiting the user cooperation. The authors analyzed the delay of a cognitive relay assisted multi-access networks, however, they did not consider the impact of primary user activities and dynamic spectrum-sharing in [12]. Most recently, some studies [13]- [17] focused on the outage performance in cognitive relay networks. 


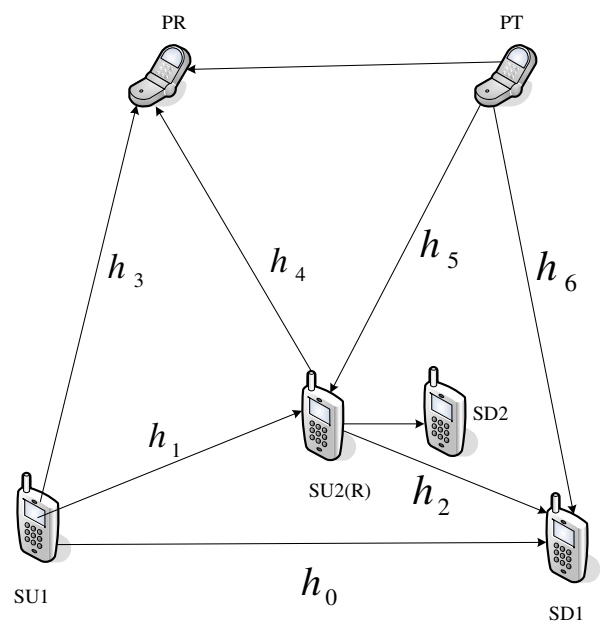

Fig. 1. System model.

The above work in cognitive relay networks has greatly improved our understanding of cognitive relay networks, such as protocol design, outage performance. However, they all have not considered the automatic-repeat-request (ARQ) [18], [19] technique in cognitive relay networks. To the best of our knowledge, the performance analysis combined cognitive relay networks and ARQ technique is almost unexplored from the analytical point view. As such, we will fill this important gap in this paper. As such, we propose two ARQ protocols in this paper, and a direct ARQ protocol for comparison.

The main contributions of this paper can be summarized as follows. First, we propose two novel ARQ protocols in cognitive relay networks, which can exploit additive transmission opportunities by cooperation with each other. Second, we derived the closed-form outage probability expressions which can provide efficient means to evaluate the effects of several parameters. Finally, we propose a new metric to evaluate the improvement of the two proposed ARQ protocols. The remained of this paper is organized as follows. In Sec. 2, the system model is presented. Followed by Sec. 3, we propose three ARQ protocols and outage probability analysis is presented. Sec. 4 provides some simulation results to validate the theory analysis. Finally, in Sec. 5, some concluding remarks are presented.

\section{System Model}

Consider a cognitive radio system with the coexistence of one primary user pair and two secondary user pairs, as depicted in Fig. 1. In the secondary network, we assume that only one pair secondary user ${ }^{1}$ can concurrently transmit with primary user. When the SD1 can not decode the packet transmitted by SU1 and SU2 can decode the packet correctly, the SU2 will participate in transmitting in next transmission round if the maximum allowable transmission round is not reached. The decode-and-forward (DF) protocol is consid- ered throughout this paper. In this paper, we considered three ARQ protocols: Cooperative ARQ I Protocol, Cooperative ARQ II Protocol, and Direct ARQ Protocol, respectively.

The Direct ARQ protocol works as follows. First, the SU1 transmits its signal to its destination SD1 in the first time round. SD1 indicates success or failure of receiving the signal by feeding back a single bit of acknowledgement (ACK) or negative-acknowledgement (NACK). If an ACK is received or the transmission rounds reaches the maximum allowed number $M$, the system stops transmitting the current message and starts transmitting a new signal. Otherwise, if a NACK is received and the transmission rounds has not reached the maximum number $M$, SU1 retransmits it. When the maximum transmission rounds are reached, SD1 still can not decode the signal, an outage is declared.

The main difference between Direct ARQ protocol and Cooperative ARQ I Protocol is that when a NACK is fed back, we should take the SU2 into consideration. If SU2 decodes the signal correctly, then at the next transmission round, SU1 and SU2 should both transmit the signal to the destination SD1. Similarly, the main difference between Direct ARQ Protocol and Cooperative ARQ II Protocol is that when a NACK is fed back, we should also take the SU2 into consideration, If SU2 decodes the signal correctly, then at the next transmission round, the better node between SU1 and SU2 will be selected to retransmit. Basically speaking, if the channel between SU1 and SD1 is in deep fading, the Cooperative ARQ I Protocol and Cooperative ARQ II Protocol will reduce the rounds of retransmission greatly.

The channel between any node is assumed Rayleigh fading throughout this paper. $h_{0}, h_{1}, h_{2}, h_{3}, h_{4}, h_{5}$, $h_{6}$ denote the channels between $\mathrm{SU} 1 \rightarrow \mathrm{SD} 1, \mathrm{SU} 1 \rightarrow \mathrm{SU} 2$, $\mathrm{SU} 2 \rightarrow \mathrm{SD} 1, \mathrm{SU} 1 \rightarrow \mathrm{PD}, \mathrm{SU} 2 \rightarrow \mathrm{PD}, \mathrm{PT} \rightarrow \mathrm{SU} 2, \mathrm{PT} \rightarrow \mathrm{SD} 1, \mathrm{re}-$ spectively. As such, the effective channel gains $\left|h_{i}\right|^{2},(i=$ $0,1, \cdots, 6)$ follow the exponential distribution with parameters $a_{i},(i=0,1, \cdots, 6)$. Therefore, the probability density function (PDF) and cumulative distribution function (CDF) of $X$, for $X \in\left\{\left|h_{0}\right|^{2},\left|h_{1}\right|^{2},\left|h_{2}\right|^{2},\left|h_{3}\right|^{2},\left|h_{4}\right|^{2},\left|h_{5}\right|^{2},\left|h_{6}\right|^{2}\right\}$, can be formulated as

$$
f_{X}(x)=\frac{1}{a} e^{-\frac{x}{a}}
$$

and

$$
F_{X}(x)=1-e^{-\frac{x}{a}}
$$

\section{Performance Analysis}

\subsection{Direct ARQ Protocol}

In this protocol, in order to satisfy the constraint of primary user, SU1 should control its transmit power. As such,

\footnotetext{
${ }^{1}$ For simplicity of presentation, the working pair of secondary users is denoted as SU1 and SD1, another pair of secondary users is represented as SU2 and SD2.
} 
the transmit power at SU1 is constrained as

$$
P_{S 1}=\frac{Q}{\left|h_{3}\right|^{2}}
$$

where $Q$ denotes the maximum allowable interference power of primary user. As such, the received signal-to-interference ratio $(\mathrm{SIR})^{2}$ can be written as

$$
\operatorname{SIR}_{S D, 1}=\frac{Q\left|h_{0}\right|^{2}}{P_{P}\left|h_{3}\right|^{2}\left|h_{6}\right|^{2}},
$$

where $P_{P}$ denotes the transmit power of primary user. According to the definition of outage in ARQ protocol, the outage probability of Direct ARQ Protocol can be mathematically derived as

$$
\begin{aligned}
P_{\text {out }}^{\text {Dir }}(L) & =\left[\operatorname{Pr}\left\{\operatorname{SIR}_{S D, 1}<\gamma\right\}\right]^{M} \\
& =\underbrace{\operatorname{Pr}\left\{\frac{Q\left|h_{0}\right|^{2}}{P_{P}\left|h_{3}\right|^{2}\left|h_{6}\right|^{2}}<\gamma\right\}}_{I_{1}}]^{M} .
\end{aligned}
$$

Therefore, the main task is to derive $I_{1} . I_{1}$ can be derived as

$$
\begin{aligned}
I_{1} & =\operatorname{Pr}\left\{\frac{Q\left|h_{0}\right|^{2}}{P_{P}\left|h_{3}\right|^{2}\left|h_{6}\right|^{2}}<\gamma\right\} \\
& =\int_{0}^{\infty} f_{\left|h_{3}\right|^{2}}(x) \int_{0}^{\infty} f_{\left|h_{6}\right|^{2}}(y) \int_{0}^{\frac{P_{P} \gamma x y}{Q}} f_{\left|h_{0}\right|^{2}}(z) d z d y d x \\
& =\int_{0}^{\infty} f_{\left|h_{3}\right|^{2}}(x) \int_{0}^{\infty} f_{\left|h_{6}\right|^{2}}(y) F_{\left|h_{0}\right|^{2}}\left(\frac{P_{P} \gamma x y}{Q}\right) d y d x \\
& =\frac{1}{a_{3} a_{6}} \int_{0}^{\infty} \int_{0}^{\infty} e^{-\frac{x}{a_{3}}} e^{-\frac{y}{a_{6}}}\left(1-e^{-\frac{P_{P} \gamma x y}{a_{0} Q}}\right) d y d x \\
& =1-\frac{1}{a_{3} a_{6}} \int_{0}^{\infty} \int_{0}^{\infty} e^{-\frac{x}{a_{3}}} e^{-\frac{y}{a_{6}}} e^{-\frac{P_{P} \gamma x y}{a_{0} Q}} d y d x \\
& =1+\frac{a_{0} Q}{a_{3} a_{6} P_{P} \gamma} e^{\frac{a_{0} Q}{a_{3} a_{6} P_{P} \gamma}} E i\left(-\frac{a_{0} Q}{a_{3} a_{6} P_{P} \gamma}\right) .
\end{aligned}
$$

In this paper, we defined a new metric - average transmission rounds to represent the goodness of the protocol. Table $1^{3}$ presents the probability that after $m$-th ${ }^{4}$ transmission round the SD1 decodes the signal successfully for Direct ARQ Protocol.

\begin{tabular}{|c|c|}
\hline Transmission Round & Probability \\
\hline 1 & $1-P_{\text {out }}^{\text {Dir }}(1)$ \\
\hline 2 & $P_{\text {out }}^{\text {Dir }}(1)-P_{\text {out }}^{\text {Dir }}(2)$ \\
\hline$\cdots$ & $\cdots$ \\
\hline$M-1$ & $P_{\text {out }}^{\text {Dir }}(M-2)-P_{\text {out }}^{\text {Dir }}(M-1)$ \\
\hline$M$ & $P_{\text {out }}^{\text {Dir }}(M-1)$ \\
\hline
\end{tabular}

Tab. 1. Probability of the considered system stop transmitting after m-th transmission round based on Direct ARQ Protocol.
As such, the average transmission rounds of Direct ARQ Protocol can be given as

$$
\begin{aligned}
T_{\text {out }}^{\text {Dir }} & =1 \times\left(1-P_{\text {out }}^{\text {Dir }}(1)\right)+2 \times\left(P_{\text {out }}^{\text {Dir }}(1)-P_{\text {out }}^{\text {Dir }}(2)\right) \\
& +\cdots+L \times P_{\text {out }}^{\text {Dir }}(L-1) \\
& =1+P_{\text {out }}^{\text {Dir }}(1)+P_{\text {out }}^{\text {Dir }}(2)+\cdots+P_{\text {out }}^{\text {Dir }}(M-1) \\
& =1+\sum_{i=1}^{M-1} P_{\text {out }}^{\text {Dir }}(i) .
\end{aligned}
$$

\subsection{Cooperative ARQ I Protocol}

Due to coexistence of SU1 and SU2 in the retransmission stage, we divided the maximum interference power $Q$ equally to SU1 and SU2 for simplicity of analysis. As such, the transmit power of SU1 and SU2 can be expressed as

$$
P_{S 1}=\frac{Q}{2\left|h_{3}\right|^{2}}
$$

and

$$
P_{S 2}=\frac{Q}{2\left|h_{4}\right|^{2}}
$$

As such, the received SIR at SD1 from SU1 and SU2 in the next transmission can be expressed as

$$
\operatorname{SIR}_{S D, 2}=\frac{Q\left|h_{0}\right|^{2}}{2 P_{P}\left|h_{3}\right|^{2}\left|h_{6}\right|^{2}},
$$

and

$$
\operatorname{SIR}_{R D, 2}=\frac{Q\left|h_{2}\right|^{2}}{2 P_{P}\left|h_{4}\right|^{2}\left|h_{6}\right|^{2}} .
$$

We define $\{T r=m\}$ denoting the event that SU2 successfully decodes the signal during the $m$-th transmission round for any $m=1,2, \cdots, M-1$. Specifically, $\{\operatorname{Tr}=M\}$ denotes the event that SU2 can not decode the signal in the first $M-1$ rounds, which means that no matter SU2 decodes successfully or not at the $M$-th transmission round, it has no chance to help in forwarding the signal. Specifically, we define $\operatorname{Pr}($ Outage $\mid \operatorname{Tr}=m)$ to represent the conditional probability that SD1 can not decode the signal after $m$ rounds given the event $\{\operatorname{Tr}=m\}$ occurred. As such, the outage probability of the considered system after the maximum transmission round can be given as

$$
P_{\text {out }}^{\text {Coop }, I}(L)=\sum_{m=1}^{M} \operatorname{Pr}\{\operatorname{Tr}=m\} \operatorname{Pr}\{\text { Outage } \mid \operatorname{Tr}=m\} .
$$

Consequently, according to the definition of $\{\mathrm{Tr}=$ $m\}(m<M)$, the probability of event $\{T r=m\}$ can be derived as (13) at the top of the following page.

\footnotetext{
${ }^{2}$ In this paper, we focus on the interference-limited scenario where the interference power from the primary user is dominant relative to the additive white Gaussian noise. As such, the additive white Gaussian noise is not considered [13].

${ }^{3}$ The probability means that $m$-th transmission round, the SD1 decodes the packet successfully. As such, the system will begin to transmit new signal.

${ }^{4}$ In the case $m=M$, no matter the system transmits successfully or not in the last transmission round, it should stop transmitting.
} 


$$
\begin{aligned}
\operatorname{Pr}\{\operatorname{Tr}=m\} & =\left[\operatorname{Pr}\left\{\frac{Q\left|h_{1}\right|^{2}}{P_{P}\left|h_{3}\right|^{2}\left|h_{5}\right|^{2}}<\gamma\right\}\right]^{m-1} \operatorname{Pr}\left\{\frac{Q\left|h_{1}\right|^{2}}{P_{P}\left|h_{3}\right|^{2}\left|h_{5}\right|^{2}}>\gamma\right\} \\
& =\left[\operatorname{Pr}\left\{\frac{Q\left|h_{1}\right|^{2}}{P_{P}\left|h_{3}\right|^{2}\left|h_{5}\right|^{2}}<\gamma\right\}\right]^{m-1}\left(1-\operatorname{Pr}\left\{\frac{Q\left|h_{1}\right|^{2}}{P_{P}\left|h_{3}\right|^{2}\left|h_{5}\right|^{2}}<\gamma\right\}\right) \\
& =-\left[1+\frac{a_{1} Q}{a_{3} a_{5} P_{P} \gamma} e^{\frac{a_{1} Q}{a_{3} P_{5} P^{\gamma} \gamma}} E i\left(-\frac{a_{1} Q}{a_{3} a_{5} P_{P} \gamma}\right)\right]^{m-1} \frac{a_{1} Q}{a_{3} a_{5} P_{P} \gamma} e^{\frac{a_{1} Q}{a_{3} a_{5} P_{P \gamma}}} E i\left(-\frac{a_{1} Q}{a_{3} a_{5} P_{P} \gamma}\right) .
\end{aligned}
$$

The conditional outage probability $\operatorname{Pr}($ Outage $\mid \operatorname{Tr}=m)$ can be derived as

$$
\begin{gathered}
P\{\text { Outage } \mid \operatorname{Tr}=m\}=[\underbrace{\operatorname{Pr}\left\{S I R_{S D, 1}<\gamma\right\}}_{I_{2}}]^{m} \\
\left.[\underbrace{\operatorname{Pr}\left\{\left(S I R_{S D, 2}+S I R_{R D, 2}\right)<\gamma\right.}_{I_{3}}\}\right]^{M-m} .
\end{gathered}
$$

The exact $I_{2}$ can be easily deduced from $I_{1}$ by substituting the parameters of $I_{1}$ with their respective counterparts. Therefore, the main focus is to derive $I_{3} . I_{3}$ can be written as

$$
\begin{aligned}
I_{3} & =\operatorname{Pr}\left\{\left(\frac{Q\left|h_{0}\right|^{2}}{2 P_{P}\left|h_{3}\right|^{2}\left|h_{6}\right|^{2}}+\frac{Q\left|h_{2}\right|^{2}}{2 P_{P}\left|h_{4}\right|^{2}\left|h_{6}\right|^{2}}\right)<\gamma\right\} \\
& =\operatorname{Pr}\left\{\left(\frac{\left|h_{0}\right|^{2}}{\left|h_{3}\right|^{2}}+\frac{\left|h_{2}\right|^{2}}{\left|h_{4}\right|^{2}}\right)<\frac{2 P_{P} \gamma\left|h_{6}\right|^{2}}{Q}\right\} .
\end{aligned}
$$

For simplicity of representation, we set $X=\frac{\left|h_{0}\right|^{2}}{\left|h_{3}\right|^{2}}, Y=$ $\frac{\left|h_{2}\right|^{2}}{\left|h_{4}\right|^{2}}$ and $Z=\frac{2 P_{P} \gamma\left|h_{6}\right|^{2}}{Q}$. As such, PDF and CDF of them can be expressed as

$$
\begin{aligned}
& F_{X}(x)= \operatorname{Pr}\{X<x\}=\operatorname{Pr}\left\{\frac{\left|h_{0}\right|^{2}}{\left|h_{3}\right|^{2}}<x\right\} \\
&= \int_{0}^{\infty} f_{\left|h_{3}\right|^{2}}(u) \int_{0}^{x u} f_{\left|h_{0}\right|^{2}}(v) d v d u \\
&= \int_{0}^{\infty} f_{\left|h_{3}\right|^{2}}(u) F_{\left|h_{0}\right|^{2}}(x u) d u \\
&= \int_{0}^{\infty} \frac{1}{a_{3}} e^{-\frac{u}{a_{3}}}\left(1-e^{-\frac{x u}{a_{0}}}\right) d u \\
&= 1-\frac{1}{a_{3}} \int_{0}^{\infty} e^{-\left(\frac{1}{a_{3}}+\frac{x}{a_{0}}\right) u} d u \\
&= \frac{a_{3} x}{a_{0}+a_{3} x}, \\
& f_{X}(x)=\frac{a_{0} a_{3}}{\left(a_{0}+a_{3} x\right)^{2}}, \\
& F_{Y}(y)=\frac{a_{4} y}{a_{2}+a_{4} y} \\
& f_{Y}(y)=\frac{a_{2} a_{4}}{\left(a_{2}+a_{4} y\right)^{2}}
\end{aligned}
$$

$$
F_{Z}(z)=1-e^{-\frac{2 P_{P} \gamma_{z}}{Q}}
$$

and

$$
f_{Z}(z)=\frac{Q}{2 P_{P} \gamma a_{6}} e^{-\frac{Q z}{2 P_{P} \gamma a_{6}}}
$$

respectively. As such, $I_{3}$ can be re-written as

$$
\begin{aligned}
I_{3} & =\operatorname{Pr}\{(X+Y)<Z\} \\
& =\int_{0}^{\infty} f_{X}(z) \int_{0}^{\infty} f_{Y}(y) \int_{x+y}^{\infty} f_{Z}(x) d z d y d x \\
& =\int_{0}^{\infty} \frac{a_{0} a_{3}}{\left(a_{0}+a_{3} x\right)^{2}} \int_{0}^{\infty} \frac{a_{2} a_{4}}{\left(a_{2}+a_{4} y\right)^{2}}\left(1-F_{Z}(x+y)\right) d y d x \\
& =\int_{0}^{\infty} \frac{a_{0} a_{3}}{\left(a_{0}+a_{3} x\right)^{2}} e^{-\frac{2 P_{P} \gamma x}{Q}} d x \int_{0}^{\infty} \frac{a_{2} a_{4}}{\left(a_{2}+a_{4} y\right)^{2}} e^{-\frac{2 P_{P} \gamma y}{Q}} d y \\
& =\frac{a_{0} a_{2}}{a_{3} a_{4}} \underbrace{\int_{0}^{\infty} \frac{1}{\left(x+\frac{a_{0}}{a_{3}}\right)^{2}} e^{-\frac{2 P_{P} \gamma x}{Q}} d x}_{I_{4}} \underbrace{\int_{0}^{\infty} \frac{1}{\left(y+\frac{a_{2}}{a_{4}}\right)^{2}} e^{-\frac{2 P_{P} \gamma y}{Q}} d y .}_{I_{5}}
\end{aligned}
$$

According to [20 (3.353.2)], we have

$$
\begin{aligned}
I_{4} & =\int_{0}^{\infty} \frac{1}{\left(x+\frac{a_{0}}{a_{3}}\right)^{2}} e^{-\frac{2 P_{P} \gamma_{x}}{Q}} d x \\
& =\frac{a_{3}}{a_{0}}+\frac{2 P_{P} \gamma}{Q} e^{\frac{2 P_{P} \gamma a_{0}}{Q a_{3}}} E i\left(-\frac{2 P_{P} \gamma a_{0}}{Q a_{3}}\right), \\
I_{5} & =\int_{0}^{\infty} \frac{1}{\left(y+\frac{a_{2}}{a_{4}}\right)^{2}} e^{-\frac{2 P_{P} \gamma y}{Q}} d y \\
& =\frac{a_{4}}{a_{2}}+\frac{2 P_{P} \gamma}{Q} e^{\frac{2 P_{P} \gamma a_{2}}{Q a_{4}}} E i\left(-\frac{2 P_{P} \gamma a_{2}}{Q a_{4}}\right) .
\end{aligned}
$$

Similarly, the probability of the event that after $m$-th transmission round the SD1 decodes the signal successfully for Cooperative ARQ I Protocol is presented in Tab. 2.

\begin{tabular}{|c|c|}
\hline Transmission Round & Probability \\
\hline 1 & $1-P_{\text {out }}^{\text {Coop }, I}(1)$ \\
\hline 2 & $P_{\text {out }}^{\text {Cop }, I}(1)-P_{\text {out }}^{\text {Coop }, I}(2)$ \\
\hline$\cdots$ & $\cdots$ \\
\hline$M-1$ & $P_{\text {out }}^{\text {Coop }, I}(M-2)-P_{\text {out }}^{\text {Coop }, I}(M-1)$ \\
\hline$M$ & $P_{\text {out }}^{\text {Coop }, I}(M-1)$ \\
\hline
\end{tabular}

Tab. 2. Probability of the considered system stop transmitting after m-th transmission round based on Cooperative ARQ I Protocol. 
As such, the average transmission rounds of Cooperative ARQ I Protocol can be given as

$$
\begin{aligned}
T_{\text {ave }}^{\text {Coop }, I} & =1 \times\left(1-P_{\text {ave }}^{\text {Coop }, I}(1)\right)+2 \times\left(P_{\text {ave }}^{\text {Coop }, I}(1)-P_{\text {ave }}^{\text {Coop }, I}(2)\right) \\
& +\cdots+M \times P_{\text {ave }}^{\text {Coop }, I}(M-1) \\
& =1+P_{\text {ave }}^{\text {Coop }, I}(1)+P_{\text {ave }}^{\text {Coop }, I}(2)+\cdots+P_{\text {ave }}^{\text {Coop }, I}(M-1) \\
& =1+\sum_{i=1}^{M-1} P_{\text {ave }}^{\text {Coop }, I}(i)
\end{aligned}
$$

Through cooperation from SU2, the additive access opportunities can be expressed as

$$
\begin{aligned}
G_{\text {Dir }}^{\text {Coop }, I} & =T_{\text {out }}^{\text {Dir }}-T_{\text {ave }}^{\text {Coop }, I} \\
& =\sum_{i=1}^{M-1}\left(P_{\text {out }}^{\text {Dir }}(i)-P_{\text {ave }}^{\text {Coop }, I}(i)\right)>0 .
\end{aligned}
$$

\subsection{Cooperative ARQ II Protocol}

Based on the Cooperative ARQ II Protocol, we find that the main difference between Cooperative ARQ II Protocol and Cooperative ARQ I Protocol is that when the SU2 can decode the signal correctly, the system selects the better node between SU1 and SU2 to retransmit. As such, if the following condition is satisfied, we will select SU2 to retransmit. Otherwise, the SU1 is selected.

$$
\frac{Q\left|h_{2}\right|^{2}}{P_{P}\left|h_{4}\right|^{2}\left|h_{6}\right|^{2}}>\frac{Q\left|h_{0}\right|^{2}}{P_{P}\left|h_{3}\right|^{2}\left|h_{6}\right|^{2}} \Rightarrow \frac{\left|h_{2}\right|^{2}}{\left|h_{4}\right|^{2}}>\frac{\left|h_{0}\right|^{2}}{\left|h_{3}\right|^{2}} .
$$

Consequently, the main difference is $I_{3}$ when we derive the outage probability of the Cooperative ARQ II Protocol. $I_{3}$ will be replaced by $I_{6}$ which is defined as follows.

$$
I_{6}=\operatorname{Pr}\left\{\max \left(S N R_{S D, 2}, S N R_{R D, 2}\right)<\gamma\right\} .
$$

Therefore, $I_{6}$ can be mathematically derived as

$$
\begin{aligned}
I_{6} & =\operatorname{Pr}\left\{\max \left(\frac{Q\left|h_{0}\right|^{2}}{P_{P}\left|h_{3}\right|^{2}\left|h_{6}\right|^{2}}, \frac{Q\left|h_{2}\right|^{2}}{P_{P}\left|h_{4}\right|^{2}\left|h_{6}\right|^{2}}\right)<\gamma\right\} \\
& =\operatorname{Pr}\left\{\frac{Q}{P_{P}\left|h_{6}\right|^{2}} \max \left(\frac{\left|h_{0}\right|^{2}}{\left|h_{3}\right|^{2}}, \frac{\left|h_{2}\right|^{2}}{\left|h_{4}\right|^{2}}\right)<\gamma\right\} \\
& =\underbrace{\operatorname{Pr}\left\{\frac{Q}{P_{P}\left|h_{6}\right|^{2}} \frac{\left|h_{0}\right|^{2}}{\left|h_{3}\right|^{2}}<\gamma\right\} \operatorname{Pr}\left\{\frac{\left|h_{0}\right|^{2}}{\left|h_{3}\right|^{2}}>\frac{\left|h_{2}\right|^{2}}{\left|h_{4}\right|^{2}}\right\}}_{I_{7}} \\
& +\underbrace{\operatorname{Pr}\left\{\frac{Q}{P_{P}\left|h_{6}\right|^{2}} \frac{\left|h_{2}\right|^{2}}{\left|h_{4}\right|^{2}}<\gamma\right\} \operatorname{Pr}\left\{\frac{\left|h_{0}\right|^{2}}{\left|h_{3}\right|^{2}}<\frac{\left|h_{2}\right|^{2}}{\left|h_{4}\right|^{2}}\right\}}_{I_{8}} .
\end{aligned}
$$

The first part of $I_{7}$ and $I_{8}$ can be easily deduced from
$I_{1}$. Therefore, another part of $I_{7}$ can be derived as

$$
\begin{aligned}
\operatorname{Pr}\{ & \left\{\frac{\left|h_{0}\right|^{2}}{\left|h_{3}\right|^{2}}>\frac{\left|h_{2}\right|^{2}}{\left|h_{4}\right|^{2}}\right\}=\operatorname{Pr}\left\{\left|h_{2}\right|^{2}<\frac{\left|h_{0}\right|^{2}\left|h_{4}\right|^{2}}{\left|h_{3}\right|^{2}}\right\} \\
& =1-\frac{1}{a_{0} a_{3} a_{4}} \int_{0}^{\infty} \int_{0}^{\infty} \int_{0}^{\infty} e^{-\frac{u}{a_{0}}} e^{-\frac{v}{a_{3}}} e^{-\frac{w}{a_{4}}} e^{-\frac{u w}{a_{2} v}} d w d v d u \\
& =1-\frac{1}{a_{4}}-\underbrace{\frac{1}{a_{0} a_{2} a_{3} a_{4}} \int_{0}^{\infty} u e^{\left(\frac{1}{a_{2} a_{3} a_{4}}-\frac{1}{a_{0}}\right) u} E i\left(-\frac{u}{a_{2} a_{3} a_{4}}\right) d u}_{I_{9}} .
\end{aligned}
$$

According to $[20(6.227 .1)]$ and after some simple mathematical manipulations, we have

$$
\begin{aligned}
I_{9} & =\frac{1}{a_{0} a_{2} a_{3} a_{4}} \int_{0}^{\infty} u e^{\left(\frac{1}{a_{2} a_{3} a_{4}}-\frac{1}{a_{0}}\right) u} E i\left(-\frac{u}{a_{2} a_{3} a_{4}}\right) d u \\
& =\theta\left(\frac{1}{\theta(\theta-1)}-\frac{1}{(\theta-1)^{2}} \ln \theta\right)
\end{aligned}
$$

where $\theta=\frac{a_{2} a_{3} a_{4}}{a_{0}}$. Similarly, the probability of event that after $m$-th transmission round the SD1 decodes the signal successfully for Cooperative ARQ II Protocol is presented in Tab. 3.

\begin{tabular}{|c|c|}
\hline Transmission Round & Probability \\
\hline 1 & $1-P_{\text {out }}^{\text {Coop }, I I}(1)$ \\
\hline 2 & $P_{\text {out }}^{\text {Cop }, I I}(1)-P_{\text {out }}^{\text {Coop }, I I}(2)$ \\
\hline$\cdots$ & $\cdots$ \\
\hline$M-1$ & $P_{\text {out }}^{\text {Coop,II }}(M-2)-P_{\text {out }}^{\text {Coop }, I I}(M-1)$ \\
\hline$M$ & $P_{\text {out }}^{\text {Coop }, I I}(M-1)$ \\
\hline
\end{tabular}

Tab. 3. Probability of the considered system stop transmitting after $m$-th transmission round based on Cooperative ARQ II Protocol.

As such, the average transmission rounds of Cooperative ARQ II Protocol can be given as

$$
T_{\text {ave }}^{\text {Coop }, I I}=1+\sum_{i=1}^{M-1} P_{\text {ave }}^{\text {Coop }, I I}(i) .
$$

Through Cooperative ARQ II Protocol, the additive access opportunities can be expressed as

$$
\begin{aligned}
G_{\text {Dir }}^{\text {Coop }, I I} & =T_{\text {out }}^{\text {Dir }}-T_{\text {ave }}^{\text {Coop }, I I} \\
& =\sum_{i=1}^{M-1}\left(P_{\text {out }}^{\text {Dir }}(i)-P_{\text {ave }}^{\text {Coop }, I I}(i)\right)>0 .
\end{aligned}
$$

Remarks: According the exact expression of the cooperation gain, we conclude that the Cooperative ARQ I Protocol and Cooperative ARQ II Protocol can achieve additive opportunities. Specifically, Cooperative ARQ II Protocol will use least energy consumption to achieve more spectrum access. However, Cooperative ARQ II Protocol needs information exchange between SU1 and SU2. 


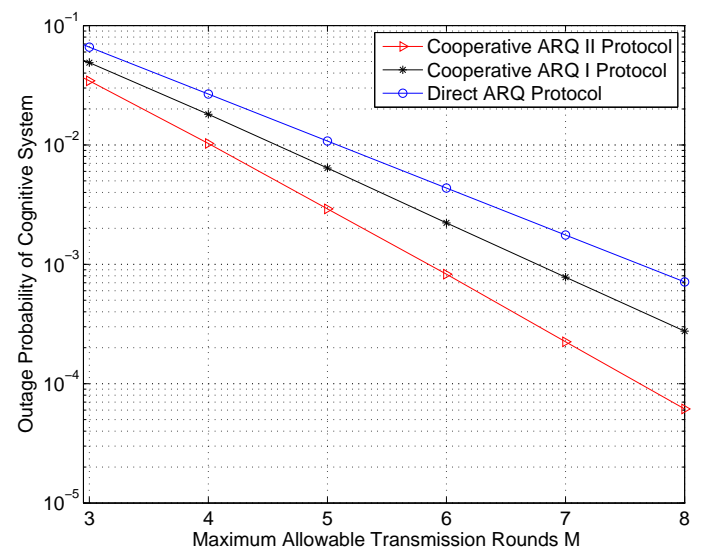

Fig. 2. Outage probability verse the maximum allowable transmission rounds when $Q=10 \mathrm{~dB}, P_{P}=10 \mathrm{~dB}$.

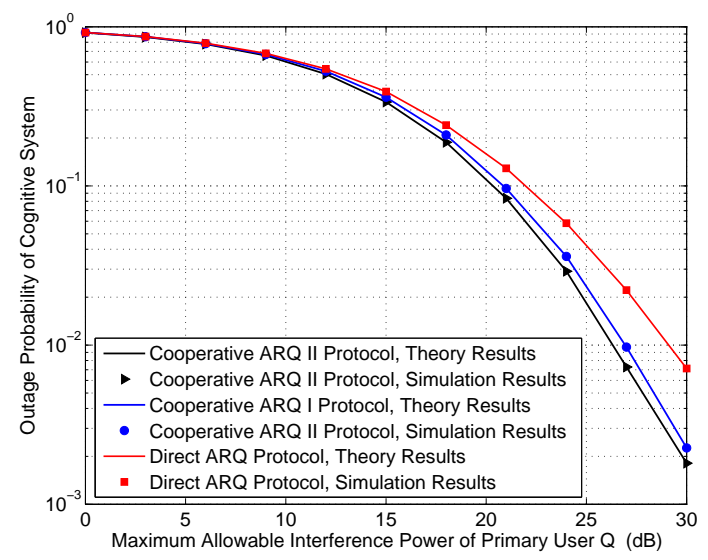

Fig. 4. Outage probability verse the maximum allowable interference power of primary user when $P_{P}=10 \mathrm{~dB}, M=10$.

\section{Numerical Results}

In this section, we provide some insight into the benefits of the proposed two ARQ-based protocols. Hereafter, we assume $a_{0}=1, a_{1}=2, a_{2}=2, a_{3}=1, a_{4}=1, a_{5}=1$, $a_{6}=1$.

Fig. 2, Fig. 3, and Fig. 4 evaluate the impact of maximum allowable transmission rounds, transmit power of primary user and maximum allowable interference power of primary user on the outage performance of considered system, respectively. From Fig. 2, we can observe that the outage probability will decrease when the maximum allowable transmission rounds increases. Obviously, the outage performance will deteriorate when transmit power of primary user increase. Similarly, the outage performance will improve when the maximum allowable interference power of primary user increases. From Fig. 2, Fig. 3, and Fig. 4, we conclude that the best performance protocol among three protocols is Cooperative ARQ II Protocol. However, in Cooperative ARQ II Protocol, information exchange between SU1 and SU2 is needed. Specifically, the theory results and the Monte Carlo simulation results match perfectly, which

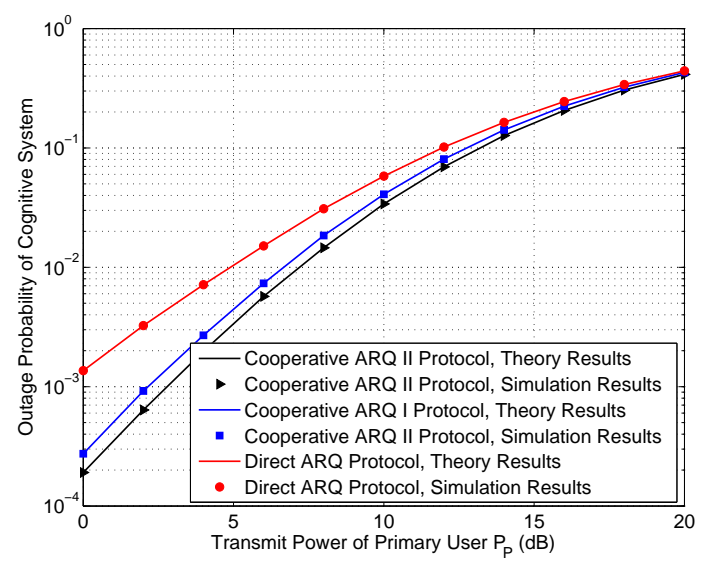

Fig. 3. Outage probability verse the transmit power of primary user when $Q=10 \mathrm{~dB}, M=10$.

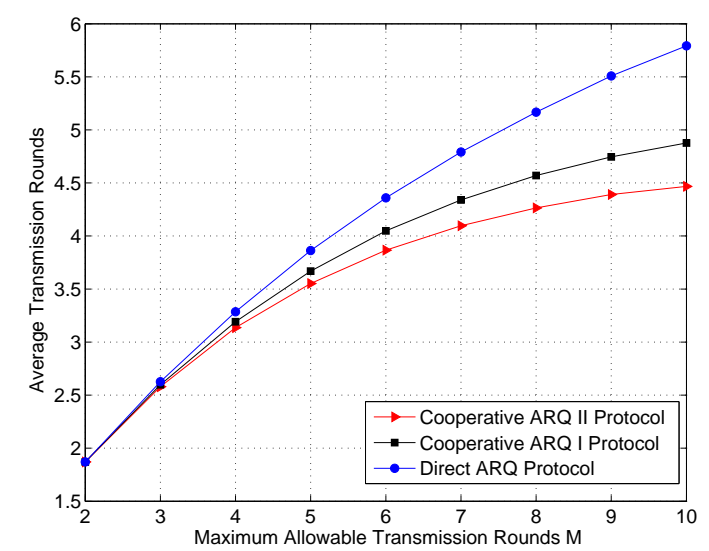

Fig. 5. Average transmission rounds verse the maximum allowable transmission rounds when $P_{P}=10 \mathrm{~dB}, Q=10 \mathrm{~dB}$.

validates the correctness of the analytical results.

Fig. 5 and Fig. 6 evaluate the average transmission rounds and additive transmission rounds gain verse the maximum allowable transmission rounds. From Fig. 5 and Fig. 6, we clearly deduce that the average transmission rounds will increase when the maximum allowable transmission rounds increases. Moreover, Fig. 6 indicates that the proposed two novel protocols can obtain some additive access opportunities by reducing the average transmission rounds of SU1 and SD1. Specifically, due to selecting the better node to transmit in retransmission stage, the energy consumption will also reduce.

Fig. 7 and Fig. 8 show the relation between average transmission rounds and transmit power of primary user, additive transmission rounds gain and transmit power of primary user, respectively. We can conclude from Fig. 7 that the average transmission rounds will increase when the transmit power of primary user increases. Interestingly, from Fig. 8, we find that the additive transmission gain is high in medium region, and low in low and high transmit power region. This can be explained as follows. When the transmit power of 


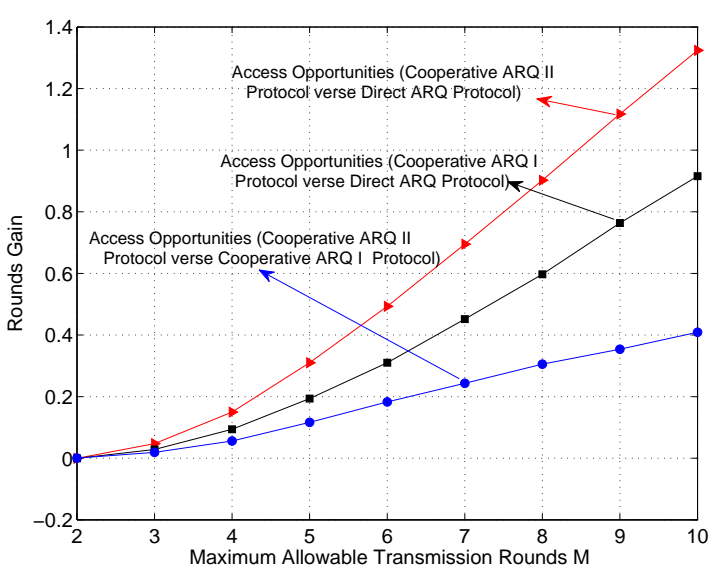

Fig. 6. Additive access opportunities verse the maximum allowable transmission rounds when $P_{P}=10 \mathrm{~dB}, Q=10 \mathrm{~dB}$.

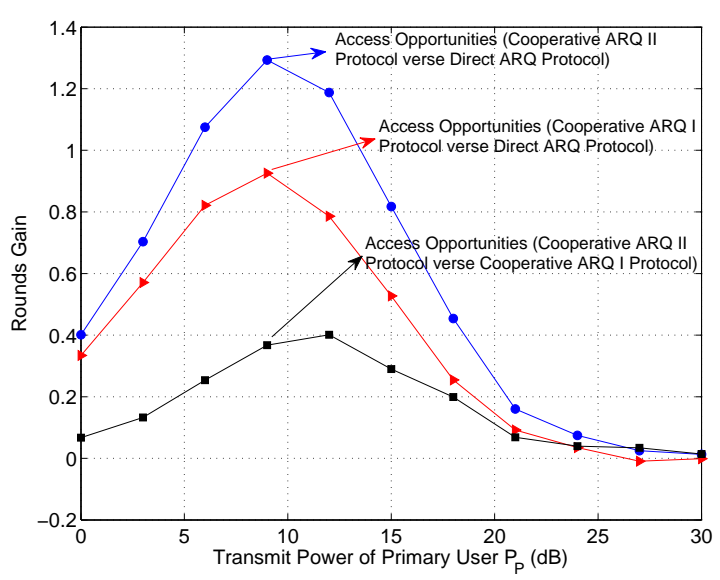

Fig. 8. Additive access opportunities verse the transmit power of primary user when $Q=10 \mathrm{~dB}, M=10$.

the primary user is in low region, SD1 will decode the signal correctly in high probability. As such, even SU2 can decode the signal successfully, it may have little cooperation opportunity in this area. In the high transmit power region, SD1 and SU2 can not decode the signal correctly with high probability. Therefore, SU2 may have no ability to forward the signal in high probability.

Fig. 9 and Fig. 10 depict the impacts of maximum allowable interference power of primary user on the average transmission rounds and additive transmission rounds gain, respectively. Contrary to the Fig. 7 and Fig. 8, the average transmission rounds will decrease when the maximum allowable interference power of primary user increases. Similarly, the additive transmission rounds gain is high in medium region of $Q$, and low in low and high of $Q$. This can be explained as follows. When the maximum allowable interference power of primary user is low, the SD1 and SU2 can not decode the signal in high probability. As a result, the SU2 has no ability to forward the signal in the retransmission stage. In high region, SD1 can decode the

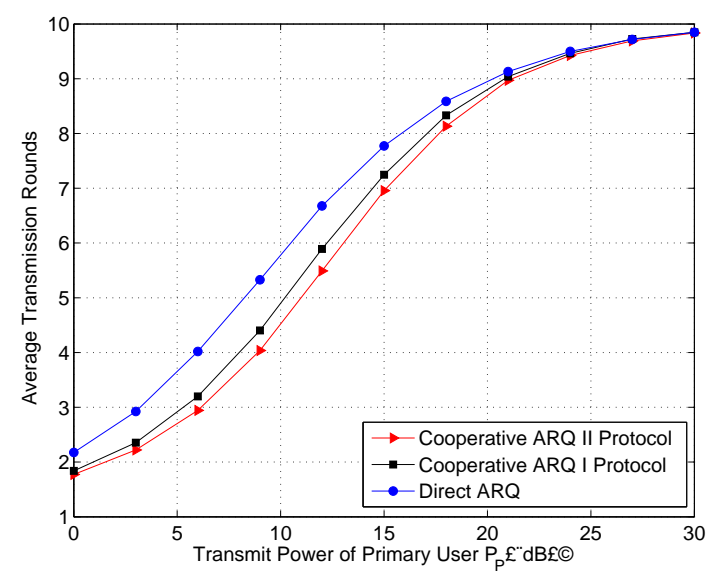

Fig. 7. Average transmission rounds verse the transmit power of primary user when $Q=10 \mathrm{~dB}, M=10$.

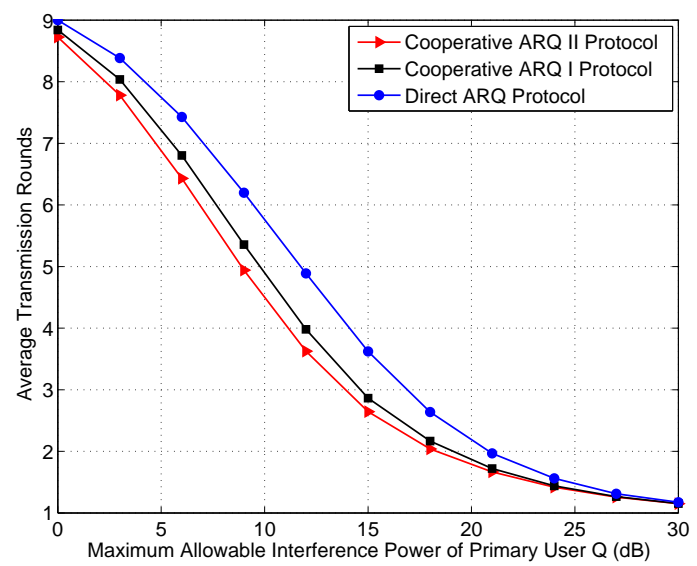

Fig. 9. Average transmission rounds verse the maximum allowable interference power of primary user when $P_{P}=10 \mathrm{~dB}$, $M=10$.

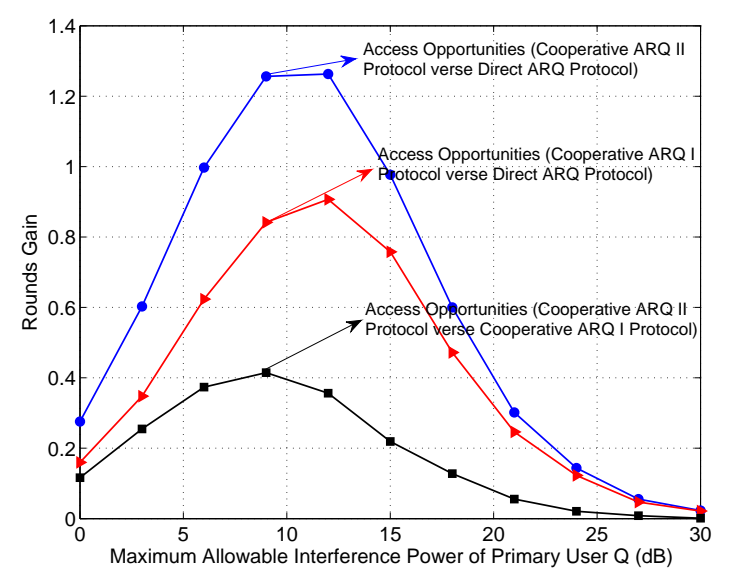

Fig. 10. Additive access opportunities verse the maximum allowable interference power of primary user when $P_{P}=$ $10 \mathrm{~dB}, M=10$.

signal successfully in high probability. As such, it needs less help from SU1 in each transmission. 


\section{Conclusion}

In this paper, we have proposed two novel ARQ-based protocols to achieve additive access opportunities in cognitive decode-and-forward relay networks. First, we have derived the closed-form outage probability expressions of the proposed protocols which provide an efficient means to evaluate the impacts of several parameters. Moreover, we define a new metric to evaluate the improvement of cooperative ARQ protocols. We can observe that, via cooperating with SU2, the outage performance and average transmission rounds can be improved greatly. As such, SU2 can obtain some access opportunities by reducing the transmission rounds of SU1. We also find that Cooperative ARQ II Protocol performs better than Cooperative ARQ I Protocol. However, there exists information exchange in Cooperative ARQ II Protocol. Finally, numerical simulation results were presented to validate the correctness of the proposed protocols.

\section{Acknowledgements}

This work was supported by the National Natural Science Foundation of China under grant no. 60932002, the National Natural Science Fund of China under grant no. 61172062, and the Natural Science Fund of Jiangsu, China under grant no. BK2011116.

\section{References}

[1] Federal Communications Commission. Spectrum Policy Task Force Report, FCC 02-155. 2002.

[2] Federal Communications Commission. Second Report and Order, FCC 08-260. 2008

[3] MITOLA, J., MAGUIRE, G. Q. Jr. Cognitive radios: making software radio more personal. IEEE Personal Communications, 1999 vol. 6 , no. 4 , p. $13-18$. DOI: $10.1109 / 98.788210$

[4] MITOLA, J. Cognitive Radio: An Integrated Agent Architecture for Software Defined Radio, Ph. D. dissertation. Stockholm (Sweden) KTH Royal Institute of Technology, 2000.

[5] XU, Y., WANG, J., WU, Q., ZHANG, Z., ANPALAGAN, A., SHEN, L. Optimal energy-efficient channel exploration for opportunistic spectrum usage. IEEE Wireless Commmunication Letters, 2012, vol. 1, no. 2, p. 77-80. DOI: 10.1109/WCL.2012.012012.110257

[6] ZHANG, Z., HUI, Q., WANG, J. Energy-efficient power allocation strategy in cognitive relay networks. Radioengineering, 2012, vol. 21 , no. 3 , p. 809-814.

[7] WU, Q., HAN, H., WANG, J., ZHAO, Z., ZHANG, Z. Sensing task allocation for heterogeneous channels in cooperative spectrum sensing. Radioengineering, 2010, vol. 19, no. 4, p. 544-551.

[8] BLETSAS, A., SHIN, H., WIN, M. Z., LIPPMAN, A. A simple cooperative diversity method based on network path selection. IEEE
Journal on Selected Areas in Communications, 2006, vol. 24, no. 3 , p. 659-672. DOI: 10.1109/JSAC.2005.862417

[9] ZHI, Q., CUI, S., SAYED, A. H. Optimal linear cooperation for spectrum sensing in cognitive radio networks. IEEE Journal of Selected Topics in Signal Processing, 2008, vol. 2, no. 1, p. 28-40. DOI: 10.1109/JSTSP.2007.914882

[10] HOU, Y. T., SHI, Y., SHERALI, H. D. Spectrum sharing for multihop networking with cognitive radios. IEEE Journal of Selected Areas in Communications, 2008, vol. 26, no. 1, p. 146-155. DOI: 10.1109/JSAC.2008.080113

[11] GANNESAN, G., LI, Y. G. Cooperative spectrum sensing in cognitive radio - part I: Two user networks. IEEE Transactions on Wireless Communications, 2007, vol. 6, no. 6, p. 2204-2213. DOI: 10.1109/TWC.2007.05775

[12] SADEK, A. K., LIU, K. J. R., EPHREMIDES, A. Cognitive multiple access via cooperation: Protocol design and performance analysis. IEEE Transactions on Information Theory, 2007, vol. 53, no. 10, p. 3677-3696. DOI: 10.1109/TIT.2007.904784

[13] YANG, P., LUO, L., QIN, J. Outage performance of cognitive relay networks with interference from primary user. IEEE Communications Letters, 2012, vol. 16, no. 10, p. 1695-1698. DOI: 10.1109/LCOMM.2012.081612.121086

[14] DUONG, T. Q., ET AL. Cognitive relay networks with multiple primary transceivers under spectrum-sharing. IEEE Signal Processing Letters, 2012, vol. 19, no. 11, p. 741-744. DOI: 10.1109/LSP.2012.2217327

[15] ZHONG, C., RATNARAJAH, T., WONG, K. K. Outage analysis of decode-and-forward cognitive dual-hop systems with the interference constraint in Nakagami- $m$ fading channels. IEEE Transactions on Vehicular Technology, 2011, vol. 60, no. 6, p. 2875-2879. DOI: 10.1109/TVT.2011.2159256

[16] DUONG, T. Q., ET AL. Outage and diversity of cognitive relaying systems under spectrum sharing environments in Nakagami- $m$ fading. IEEE Communications Letters, 2012, vol. 16, no. 12, p. 20752078. DOI: 10.1109/LCOMM.2012.100812.121859

[17] DUONG, T. Q., DA COSTA, D. B., ELKASHLAN, M., BAO, V. N. Q. Cognitive amplify-and-forward relay networks over Nakagami-m fading. IEEE Transactions on Vehicular Technology, 2012, vol. 61, no. 5, p. 2368-2374. DOI: 10.1109/TVT.2012.2192509

[18] TANNIOUS, R., NOSRATINIA, A. Coexistence through ARQ restransmission in fading cognitive radio channels. In IEEE International Symposium on Information Theory Proceedings. Austin (TX, USA), 2010, p. 2078-2082. DOI: 10.1109/ISIT.2010.5513394

[19] GUAN, X., CAI, Y., SHENG, Y., YANG, W. Exploiting primary retransmission to improve secondary throughput by cognitive relaying with best-relay selection. IET Communications, 2012, vol. 6, no. 2, p. 1769-1780. DOI: 10.1049 /iet-com.2011.0940

[20] GRADSHTEYN, I. S., RYZHIK, I. M. Table of Integrals, Series, and Products, 5th ed. Orlando (FL, USA): Academic Press, 1994.

\section{About the Authors...}

Zongsheng ZHANG was born in 1986. He received his B.S. degree in Communications Engineering from Institute of Communications, Nanjing, China, in 2009. He is currently pursuing the Ph.D. degree in Communications and Information Systems from Institute of Communications, PLA University of Science and Technology. His research interests focus on wireless communications, cognitive radio and channel coding techniques. 
Jinlong WANG was born in 1963. He received his B.S. degree in Wireless Communications, M.S. degree and Ph.D. degree in Communications and Electronic Systems from Institute of Communications Engineering, Nanjing, China, in 1983, 1986 and 1992, respectively. He is currently professor at the PLA University of Science and Technology, China. $\mathrm{He}$ is also the co-chairman of IEEE Nanjing Section. He has published widely in the areas of signal processing for communications, information theory, and wireless networks. His current research interests include wireless communication, cognitive radio, soft-defined radio and ultra-wide bandwidth
(UWB) systems.

Qihui WU was born in 1970. He received his B.S. degree in Communications Engineering, M.S. degree and Ph.D. degree in Communications and Information Systems from Institute of Communications Engineering, Nanjing, China, in 1994, 1997 and 2000, respectively. He is currently professor at the PLA University of Science and Technology, China. His current research interests are algorithms and optimization for cognitive wireless networks, soft-defined radio and wireless communication. 